\title{
Development of a Point Tracking System for Measuring Structural Deformations Using Commercial Video Cameras
}

\author{
Hong-Il Kim*, Ho-Young Kim**, Hyun-Jin Park** and Jae-Hung Han****** \\ Department of Aerospace Engineering, \\ Korea Advanced Institude of Science and Technology \\ Dajeon, Republic of Korea \\ Jun-Bum Kim*** \\ Department of Mechanical Engineering, \\ Korea Advanced Institude of Science and Technology \\ Dajeon, Republic of Korea \\ Do-Hyung Kim**** \\ Korea Aerospace Research Institude, Daejeon, Korea
}

Jeongho Han*

Agency for Defense Development, Daejeon, Korea

\begin{abstract}
This paper deals with the creation of a new, low-cost point/position tracking system that can measure deformations in engineering structures with simple commercially widespread cameras. Though point tracking systems do exist today, such as Stereo Pattern Recognition (SPR) and Projection Moiré Interferometry (PMI) systems, they are far too costly to use to analyze small, simple structures because complex optical components such as large flashes, high-resolution cameras and data acquisition systems with several computers are required. We developed a point tracking system using commercial cameras. This system used IR LEDs and commercial IR CCD cameras to minimize the interference posed by other extraneous light sources. The main algorithm used for this system is an optical point tracking algorithm, which is composed of the point extraction algorithm and the point matching algorithm for $3-\mathrm{D}$ motion estimation. a series of verification tests were performed. Then, the developed point tracking system was applied to measure deformations of an acrylic plate under a mechanical load. The measured deformations of the acrylic plate matched well with the numerical analysis results. The results indicate that the developed point tracking system is reliable enough to measure continuous deformed shapes of various engineering structures.
\end{abstract}

Key words : 3-D position estimation algorithm, Specific point extraction algorithm, SPR (Stereo Pattern Recognition), Commercial cameras

\footnotetext{
* Graduate Student

** Undergraduate Student

*** Undergraduate Student

**** Director

***** Director

****** Professor

E-mail : jaehunghan@kaist.ac.kr Tel : +82-42-350-3723 Fax : +82-42-350-3710
} 


\section{Introduction}

One of the emerging issues in structure technology is the estimation of structural shape changes. Especially in the case of the aerospace structures, which change shapes continuously while interacting with aerodynamic forces, it is necessary to acquire the exact shapes of the structures to understand the roles of structural flexibility and to enhance the aerodynamics and stability of the structure itself. Thus, several technologies based on optics have been introduced to the aerospace engineering field for shape measurement of 'wing' structures. Typical shape measurement techniques such as PGM (Projected Grid Method), PMI (Projection Moiré Interferometry) and SPR (Stereo Pattern Recognition) have been used in several wind tunnel tests. Van der Draai et al [1] described two different Moiré techniques such as the Painted Pattern Method (PaPM) and the Projection Pattern Method (PrPM) which tracked the motion of specific points placed on the structure surface for the deformation measurements of a dummy wing. Fleming et al [2-3] used PMI to obtain near instantaneous helicopter blade deformations for HART-I (Higher harmonic control Aeroacoustic Rotor Test). PMI was also applied to measure the structural deformation of MAV wings during a series of wind tunnel tests as shown in Fig.1. Although PMI is very good in measurement uncertainty, it is very difficult to apply the PMI method for helicopter blades and MAV wings whose deformations include rigid body motions such as flapping and leadlag, which might induce misalignment. Therefore, the SPR (Stereo Pattern Recognition) method was recently introduced to measure wing motions. Schneider [4] applied the SPR method, where 3-D positions of several markers were traced, to rotating blades for the HART-II project. The measurement uncertainties of SPR were reported to be under $0.5 \mathrm{~mm}$ and around $0.5^{\circ}$ for measuring the motions of blades of $2 \mathrm{~m}$ radius. The described systems, however, require complex optical components such as large flashes, high-resolution cameras and data acquisition systems with several computers. These systems are very large and costly to perform wind-tunnel tests of small scale structures. Thus, in this study, a point tracking system was developed using four commercial CCDs and IR LEDs. The software composed of a specific point extraction algorithm and a specific point matching algorithm, which allowed the calculation of 3-D coordinates, was developed. After configuring the point tracking system, a series of tests were performed to evaluate the system' s performance. Then, the developed point tracking system was applied to measure the deformations of an acrylic plate under a mechanical load. By comparing the acquired deformations with the results from numerical analysis, the developed point tracking system was verified.

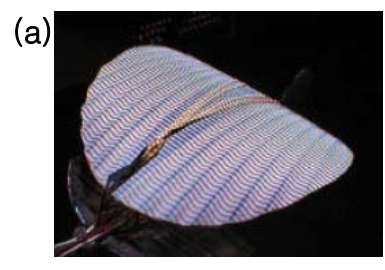

(b)

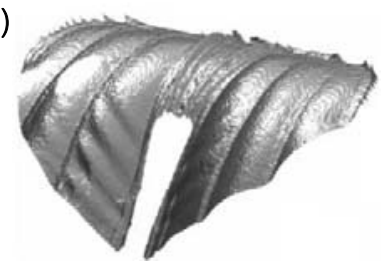

Fig. 1. (a) MAV with PMI projection grid lines [3]

(b) 3-D representation of MAV wind shapes measured by PMI [3]

\section{The Principle of 3-D Point Tracking}

By using multiple cameras, the $3-\mathrm{D}$ coordinates of the points are precisely obtained using a specific point extraction algorithm and a $3-D$ position estimation algorithm in sequence with camera calibration data as in Fig. 2. 


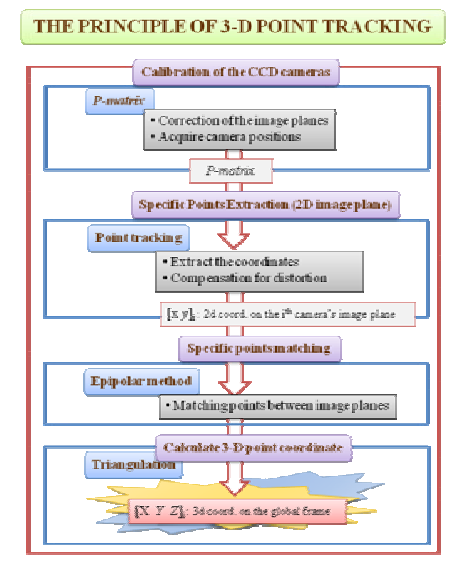

Fig. 2. The calculation process of $3-D$ point tracking

\subsection{Calibration of the CCD cameras}

The camera is the apparatus which maps the $3-\mathrm{D}$ real world $(X, Y, Z)^{\mathrm{T}}$ into a $2-\mathrm{D}$ image $(x, y)^{\mathrm{T}}$ as expressed in eq. (1). The mapping characteristics of each camera are contained in camera projection matrices $(P$-matrices). In the case of a general camera projection matrix: $P-$ matrices are composed of a camera calibration matrix $K_{3 \times 3}$, which contains the intrinsic parameters of the camera, a rotation matrix $R_{3 \times 3}$, and a translation matrix $T_{3 \times 1}$. The rotation and translation matrices contain the extrinsic parameters between cameras as eq. (2). For a real CCD camera, the $P$-matrix is expressed using a new camera calibration matrix $K$ as shown in eq. (3).

$$
\begin{gathered}
(X, Y, Z)^{T} \Rightarrow(x, y)^{T} \\
P=K[R \mid t] \\
\hat{x}=\left[\begin{array}{ccc}
\alpha_{x} & s & x_{0} \\
& \alpha_{y} & y_{0} \\
& & 1
\end{array}\right][R \mid t]\left(\begin{array}{c}
X \\
Y \\
Z \\
1
\end{array}\right)
\end{gathered}
$$

where $\alpha_{x}, \alpha_{y}$ are the focal lengths in pixel dimensions, $m_{x}, m_{y}$ are the number of pixels per unit distance along the $x$ and $y$ direction, $x_{0}, y_{0}$ are the position of the principal points in pixel dimensions and $s$ is the skew parameter. This $P$-matrix including $K, R$ and $t$ matrices of the CCD used in this study, were obtained using the Camera Calibration Toolbox in MATLAB [5].

\subsection{Specific Points Extraction}

In this step, the $2-\mathrm{D}$ coordinates of the specific points are obtained in pixel dimensions in terms of each camera. We used IR illumination and attached reflective markers on the structure surfaces to easily distinguish the markers because the markers appear much brighter than the rest of the image. The exact positions of the markers, the center positions of the brighter spots, are calculated using the Gaussian mask method as shown in Fig. 3.

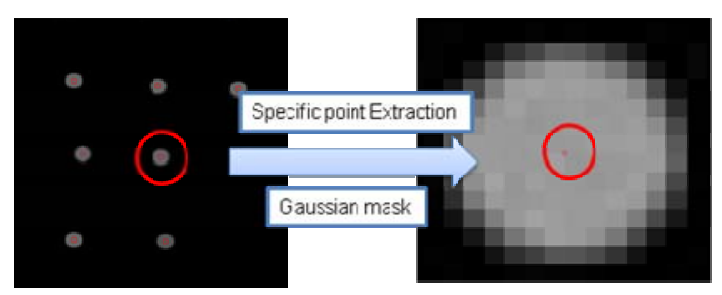

Fig. 3. Specific point (Marker reflection) extraction 


\subsection{Specific points matching using epipolar geometry}

Before calculating the $3-\mathrm{D}$ coordinates of specific points, the $2-\mathrm{D}$ coordinates of random points in image planes of cameras should be matched each other. Usually, the epipolar constraint is used to perform the specific points matching. Because the epipolar constraint implies that the point of interest and the projected points of multiple cameras should lie on the same plane (epipolar plane in Fig. 4), we can obtain matched specific points in $2-\mathrm{D}$ coordinate of image planes from the epipolar constraint. The epipolar constraint is expressed in eq. (4) using camera rotation matrix $R$ and translation matrix $t$.

$$
P_{1}^{T} R[t]_{X} P_{2}=0
$$

where $P_{1}$ and $P_{2}$ represent the specific points on the image planes and []$_{\mathrm{x}}$ represents the cross product.

\subsection{Calculation of 3-D coordinates of matched points}

Generally, the concept of triangulation is applied to calculate the 3-D coordinates of the matched points. Theoretically, the back-project rays should meet at one point ( $G$ point in Fig. 4), but errors always exist. Thus, the point whose $3-\mathrm{D}$ position is closest to backproject rays of specific points in image planes is obtained as depicted in Fig. 5. The optimum point is searched for by minimizing the total distance $D$.

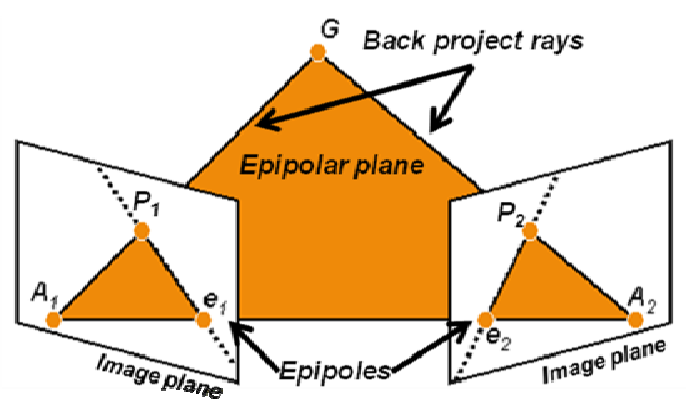

Fig. 4. Epipolar geometry [6]

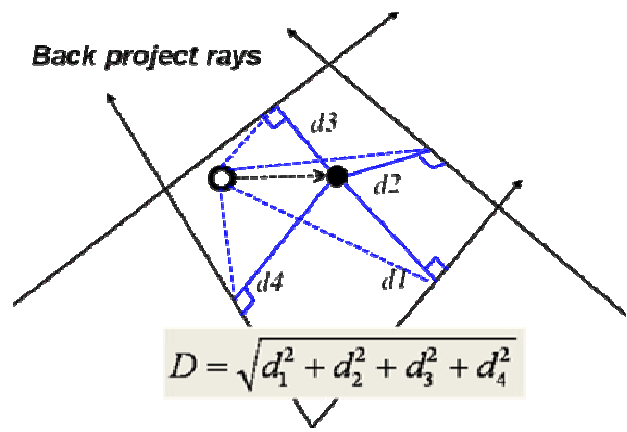

Fig. 5. Calculation of 3-D coordinates of specific points

\section{System Preparation}

We prepared a point tracking system using four-CCD cameras with a single personal computer for enhancing the point track accuracy. IR LEDs were attached to the CCD cameras for illumination, and CCD cameras were modified to detect only IR light, because it is more convenient and easier to detect IR light than visible light in normal room conditions.

\subsection{System configuration}

Commercially available webcams (Microsoft VX-3000) were used for the CCD camera system. Since the cameras are low-cost (\$39.95), they have limited capability: 640 x 480 pixel resolution, data collection rate of 30 frames per second, and a USB 1.1 connection. Their size $(53 \mathrm{~mm} \times 65 \mathrm{~mm})$ is small, like any common webcam on the market. However, the VX-3000 cameras cannot detect infrared light. This is due to filters placed on the lens, which prevent color distortion when displaying images with only the visible light spectrum. Therefore, the IR filters were removed and replaced with a visible light filter to capture only IR light, cut to fit the size of the camera lens that can block visible light, but not infrared light. 
For the cameras to be capable of detecting infrared light there has to be a source that reflects light off of the numerous markers located on the structure of interest. Hence, the source was six LED infrared diodes (AUX SI3317-H) with a range of $850 \mathrm{~mm}$ soldered onto a stripboard $(80 \mathrm{~mm} \times 80 \mathrm{~mm})$, which was then attached to the front of the cameras. Electricity was supplied through the means of a power amplifier.

Four cameras were then placed on tripods, which were then glued (with a glue gun) onto an acrylic plate $(300 \mathrm{~mm} \times 200 \mathrm{~mm}$ ) different from the one under analysis. Fig. 6 shows how the system looks like.

\subsection{Software development}

As mentioned before, the software used in cameras is composed of two parts: the first part computes 2-D dimensional pixel data and the second part calculates the $3-\mathrm{D}$ coordinates. The program uses a 'Specific point extraction algorithm' to extract the points reflected from the markers to subsequently find the $2-\mathrm{D}$ pixel coordinates of the points of interest on the image plane, and also corrects for spherical aberrations. The 2-D pixel coordinates, if within an error range, are grouped with coordinate values that were recorded in a similar time frame then analyzed through a 'Specific point matching algorithm' and

'Triangulation'. The final results are the $3-\mathrm{D}$ coordinates of the points. Fig. 7 is the picture of the developed software in action.

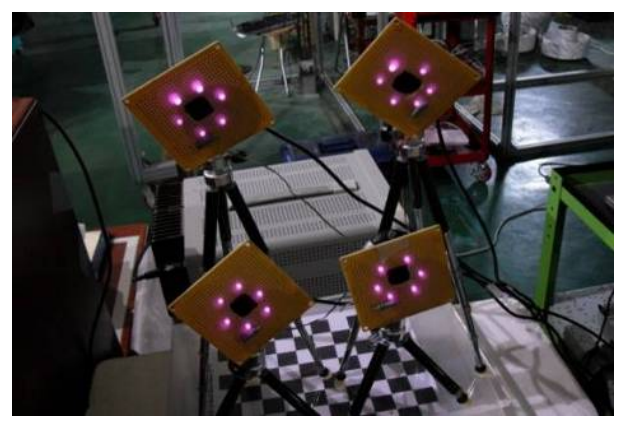

Fig. 6. VX-3000 webcams with IR LEDs

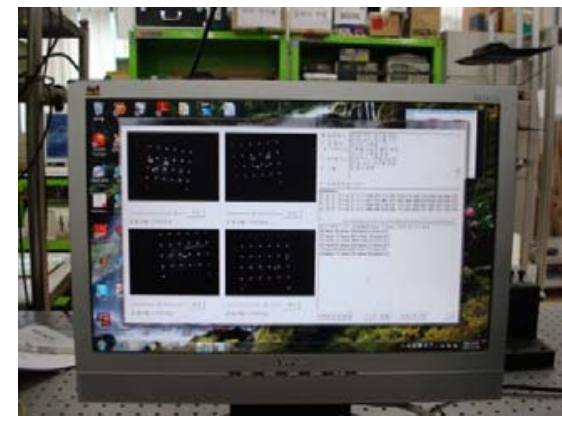

Fig. 7. Screenshot of the developed software in action

\subsection{System verification test}

A series of verification tests were performed in order to evaluate the uncertainties of the system. For $3-\mathrm{D}$ point tracking, both the orthogonal and parallel directions to the image plane were measured successfully as depicted in Fig. 8. Thus, in this verification test, we prepared vernier calipers and micrometers to simulate parallel and orthogonal motion with areflective marker, respectively. A vernier caliper and micrometer were set on a stationary clamp so that one end could slide out. A small section of reflective paper was affixed to the movable ends. This is shown in Fig. 9. The caliper and micrometer were placed in front of the camera system, so the reflective paper could be moved by exact measures. Fig. 10 shows the measured displacement when the end of the caliper traveled $1 \mathrm{~mm}, 10 \mathrm{~mm}$ and $20 \mathrm{~mm}$ along the orthogonal direction. Fig. 11 shows the histograms of the measured displacement of the caliper which traveled $1 \mathrm{~mm}, 10 \mathrm{~mm}$ and $20 \mathrm{~mm}$ along the parallel direction. From the results, the parallel and orthogonal movements of the reflective marker were successfully estimated using the newly developed point tracking system.

For a quantitative aspect, the errors and standard deviations are presented in Table 1. From the table, the maximum error is $0.34 \mathrm{~mm}$ and maximum standard deviation is $0.36 \mathrm{~mm}$. These results imply that the point tracking system might ensure a measurement uncertainty under $0.5 \mathrm{~mm}$. Compared to the SPR system used in the HART-II program, whose resolution is $0.4 \mathrm{~mm}$, its performance is remarkable. Therefore, the developed point tracking system could be used to measure deformations in structures. 


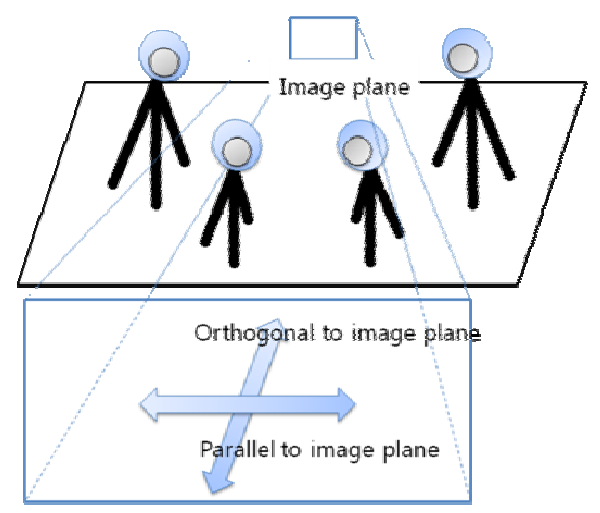

Fig. 8. Verification for orthogonal and parallel directions

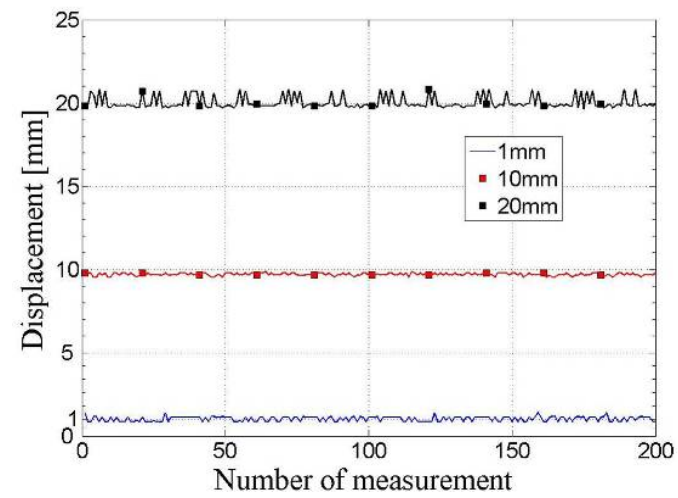

Fig. 10. Measurement results for when the reflective marker travels in the orthogonal direction

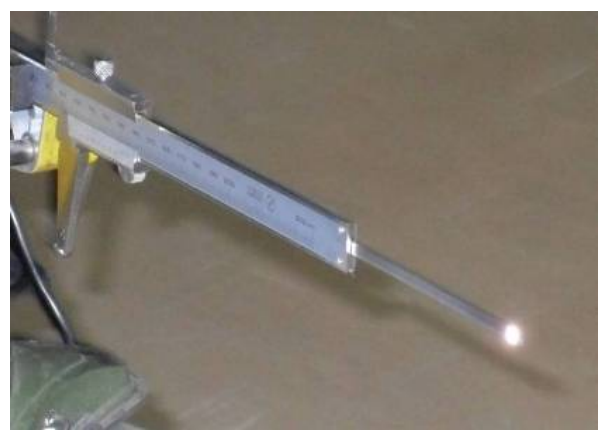

Fig. 9. A vernier caliper with reflective paper attached for parallel direction test

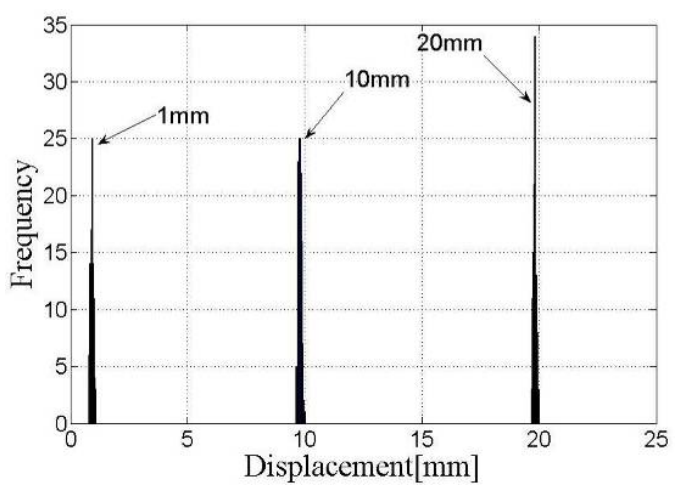

Fig. 11. Histograms for when the reflective marker travels in the parallel direction

Table 1. Measurement results: errors \& standard deviations

\begin{tabular}{c|c|c|c}
\hline \hline & Travel $[\mathrm{mm}]$ & Errors $[\mathrm{mm}]$ & STD $[\mathrm{mm}]$ \\
\hline \hline \multirow{3}{*}{ Parallel direction } & 1.00 & -0.07 & 0.13 \\
\cline { 2 - 4 } & 10.00 & -0.21 & 0.16 \\
\cline { 2 - 4 } & 20.00 & -0.18 & 0.13 \\
\hline \multirow{3}{*}{$\begin{array}{c}\text { Orthogonal } \\
\text { direction }\end{array}$} & 1.00 & 0.12 & 0.18 \\
\cline { 2 - 4 } & 10.00 & -0.34 & 0.12 \\
\cline { 2 - 4 } & 20.00 & -0.18 & 0.36 \\
\hline \hline
\end{tabular}

\section{Experiments}

The point tracking system was used to measure the deformation of an acrylic plate in static conditions. Various masses were hanged to the end of the plate, which yielded numerous analyzable cases.

\subsection{Experimental setup}

The plate on which the cameras were attached was placed horizontally so the cameras could look downwards in the negative $z$ direction (refer to Fig. 12 for a conceptual diagram). The 


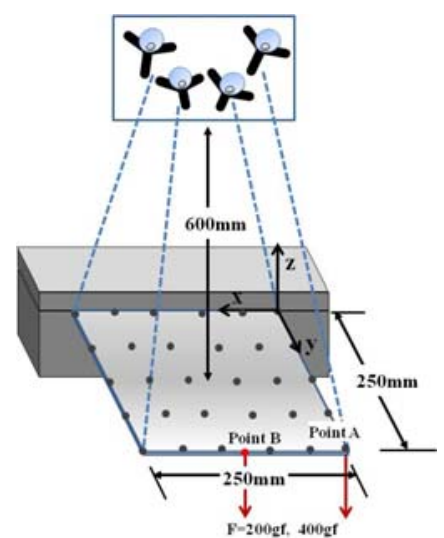

Fig. 12. Experimental setup for measuring the acrylic plate

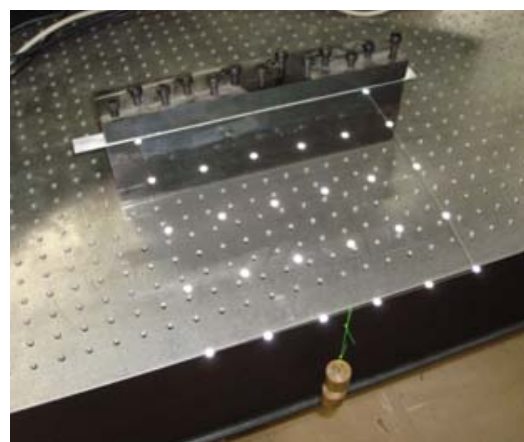

Fig. 13. Reflective markers on the acrylic plate surface and masses hanged in the center of plate edge (point B)

structure under analysis is an acrylic plate $(250 \mathrm{~mm} \times 250 \mathrm{~mm})$, with infrared reflecting markers placed in a 6 by 5 pattern. As mentioned, the plates were clamped on one end, the placed approximately $600 \mathrm{~mm}$ from the camera system. Two holes were also made, at point A and point B that allowed for varying masses to be hanged, which cause the deformation of the plate (Fig. 13).

\subsection{Experiments}

The experiments were executed for measuring plate deformation by varying the masses used, and the points where masses would be hung. In this paper, the results for four conditions are reported. The first set involved $200 \mathrm{~g}$ and $400 \mathrm{~g}$ of mass hung from a corner of the plate (point A in Fig. 12), and the according deformations were measured. The second set measured deformations caused by the same masses attached to the center of the plate' s edge (point B in Fig. 12).

\subsection{Results}

In this section, the measured plate deformations are plotted for all conditions and such deformations are shown in Fig. 14. All The results show that as more mass and thus more stress was applied to the plate, it continued to bend in the negative $z$ direction. With $200 \mathrm{~g}$ attached, the magnitude of bending at point A reached around a maximum of $37.2 \mathrm{~mm}$ compared to the $400 \mathrm{~g}$ case, which was $66.6 \mathrm{~mm}$. This was of course when the weights end up placed at a corner, point A. When hung at point $\mathrm{B}$, for $200 \mathrm{~g}$, the plate deforms by $26.4 \mathrm{~mm}$. The deformation is much more pronounced at $400 \mathrm{~g}$, and deforms by almost $51 \mathrm{~mm}$. From these results, it can be stated that the deformation is greater when the weights are hung from point $\mathrm{A}$ than point $\mathrm{B}$.

To verify that the results found are indeed similar to real-world phenomena, a comparison was made with values taken from an analysis program Ansys. The following values were used to simulate experimental conditions, length of plate: $250 \mathrm{~mm}$, width of plate: $250 \mathrm{~mm}$, thickness of plate: $0.0017 \mathrm{~m}$, plate density: $2,000 \mathrm{~kg} / \mathrm{m}^{3}$, and Young' $\mathrm{s}$ modulus: $3.9 \mathrm{GPa}$. Gravity was of course, also taken into account. In order to start the simulation, force was applied to a single node on the structure along the negative $z$ direction. The obtained shapes of the plate are shown in Fig. 14 on the right side of the experimental results. From Fig. 14, it was observed that the overall shapes and magnitudes of the plate acquired from experiments are very similar to those received from numerical analysis.

To quantitatively evaluate the experimental results, the MAC (Modal Assurance Criterion) value, which shows how similar the shapes are to their simulated counterparts, was introduced (eq. (5)). 


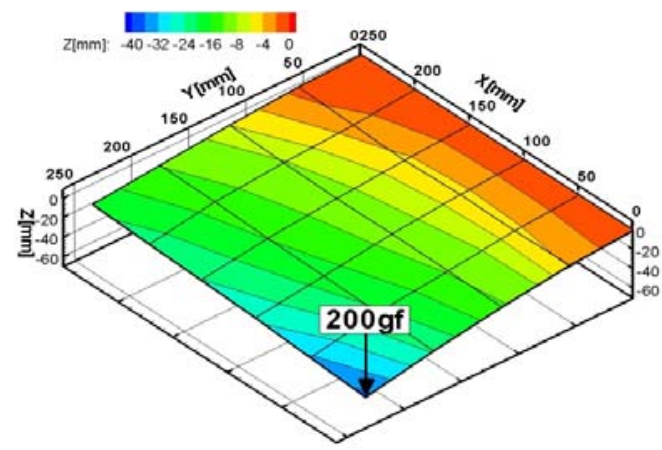

(a) Experimental results : corner mass of $200 \mathrm{~g}$

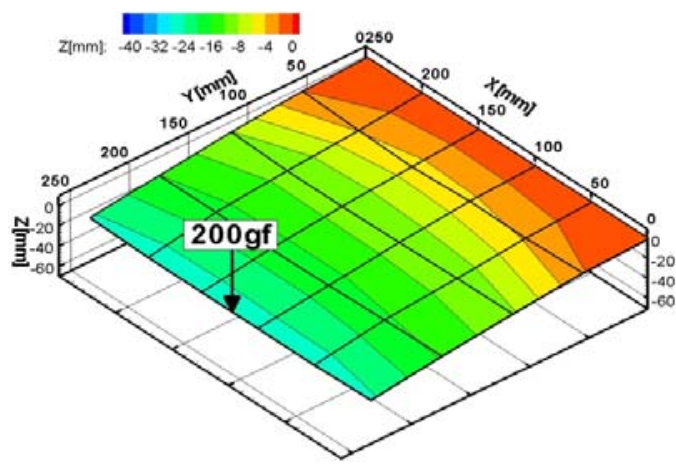

(c) Experimental results : center mass of $200 \mathrm{~g}$

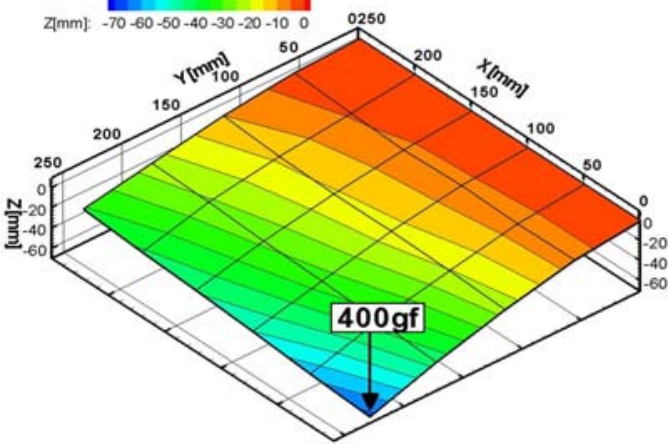

(e) Experimental results : corner mass of $400 \mathrm{~g}$

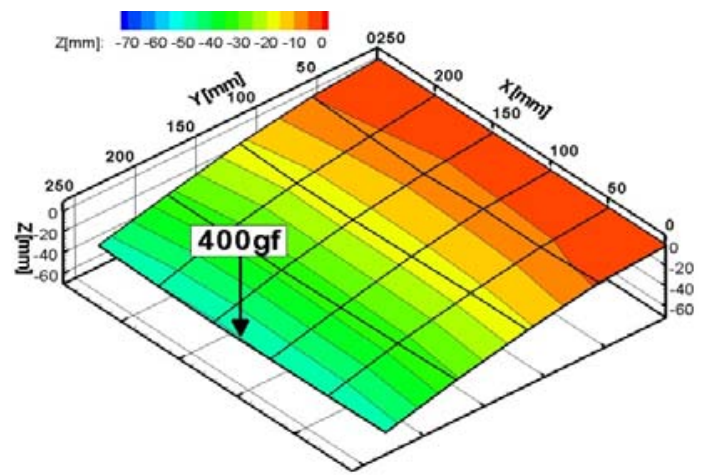

(g) Experimental results : center mass of $400 \mathrm{~g}$

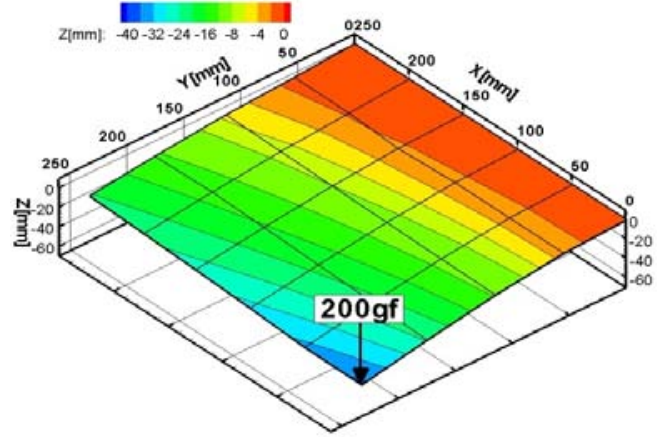

b) Analysis results : corner mass of $200 \mathrm{~g}$

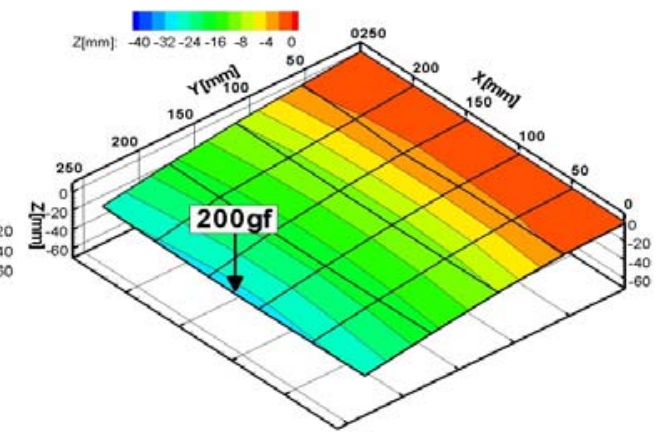

(d) Analysis results : center mass of $200 \mathrm{~g}$

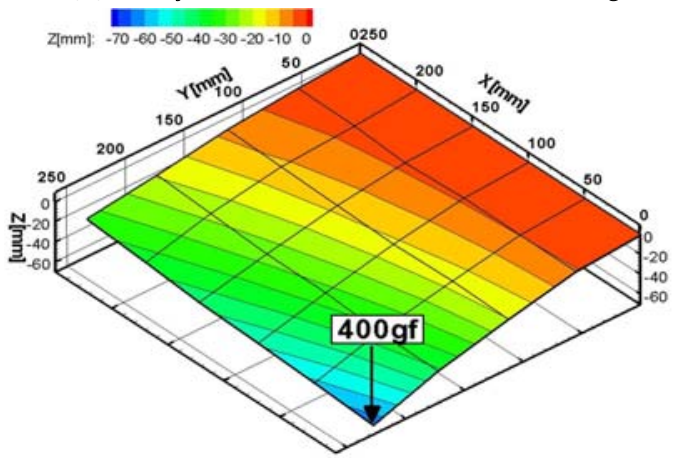

(f) Analysis results : corner mass of $400 \mathrm{~g}$

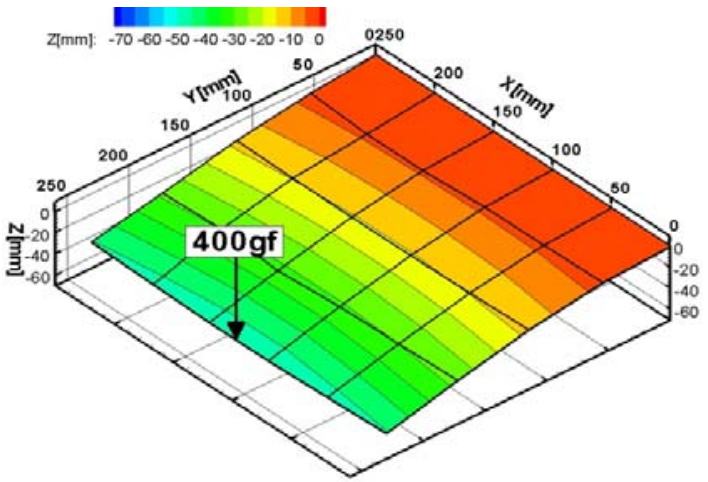

(h) Analysis results : center mass of $400 \mathrm{~g}$

Fig. 14. Deformed shape of the acrylic plate:

(left sides) Experimental results, (right sides) Analysis results 
Table 2. Test conditions and MAC values

\begin{tabular}{c|c|c|}
\hline \hline Mass location & Mass & MAC \\
\hline \multirow{2}{*}{ Corner (Point A) } & $200 \mathrm{~g}$ & 0.9959 \\
\cline { 2 - 3 } & $400 \mathrm{~g}$ & 0.9982 \\
\hline \multirow{2}{*}{ Center (Point B) } & $200 \mathrm{~g}$ & 0.9944 \\
\cline { 2 - 3 } & $400 \mathrm{~g}$ & 0.9979 \\
\hline \hline \\
$\qquad A C=\frac{\left|\phi_{E}{ }^{T} \phi_{A}\right|^{2}}{\left(\phi_{E}{ }^{T} \phi_{E}\right)\left(\phi_{A}{ }^{T} \phi_{A}\right)}$
\end{tabular}

Where is the deformation measured by the developed point tracking system and is the deformation value found by numerical analysis.

The MAC values for each case are presented in table 2. For all the cases, the MAC values were very close to the 1 . These results confirm that measured shapes of the plate are very similar to the shapes obtained in numerical analysis. As a result, it is clear that the developed point tracking system using commercial cameras are sufficiently reliable to measure continuous deformed shapes of many engineering structures.

\section{Conclusion}

In this study, we developed a point tracking system using commercial cameras. Four commercial IR CCD cameras were prepared with IR LEDs to track the markers in the IR band to avoid any interference posed by visible light. The measurement software was developed based on the specific point extraction method and point matching algorithm with a $3-\mathrm{D}$ coordinate calculation method. From a series of verification tests, the performance of the developed system was evaluated: the uncertainty in point tracking has shown to be \pm $0.5 \mathrm{~mm}$ from distances around $600 \mathrm{~mm}$. Consequently, we applied the point tracking system to quantify the deformed shape of the acrylic plate. As the results show, the obtained shapes are quite similar, showing concrete proof that the system created is reliable to an extent. The present point tracking system provides a very economical but powerful solution to measure continuous deformed shapes of many engineering structures.

\section{Acknowledgments}

This study has been supported by KARI under the KHP Dual-Use Component Development Program funded by the Ministry of Knowledge Economy.

\section{References}

1. R. K. van der Draai, R. P. M. van Schinkel and A. Telesca, "A new approach to measuring model deflection", 18th International Congress on Instrumen- tation in Aerospace Simulation Facilities, pp. 33/1 pp. 33/7, 1999

2. G. A. Fleming and S. A. Gorton, "Measurement of Rotorcraft Blade Deformation using Projection Moiré Interferometry” , Proceedings of SPIE, Vol. 3411, pp. 514-527, 1998

3. G. A. Fleming, S. M. Bartman, M. R. Waszak and L. N. Jenkins, "Projection moiré interferometry measurements of micro air vehicle wings" , Proceedings of SPIE, Vol. 4448, pp. 90-101, 2001

4. O. Schneider, "Analysis of SPR measurements form HART II" , Aerospace Science and Technology, Vol. 9, pp. 409-420, 2005

5. Camera Calibration Toolbox for MATLAB, http://www. vision.caltech.edu/bouguetj/calib_doc/

6. R. Hartly and A. Zesserman, Multiple View Geometry in Computer Vision, Cambridge Press, 2003. 